\title{
A Case of Smoldering Multiple Myeloma with Simultaneous Increase of a Polyclonal Immunoglobulin
}

\author{
Anwarul Islam ${ }^{1 *}$ and Joanne Becker ${ }^{2}$ \\ ${ }^{1}$ Clinical Associate Professor of Medicine, Division of Hematology/Oncology, Department of Medicine, Buffalo General Hospital, \\ Buffalo, New York 14203, USA
}

${ }^{2}$ Associate Professor and Clinical Chief, Division of Laboratory Medicine, Department of Pathology and Laboratory Medicine, Medical Director, Blood Bank and Therapeutic Apheresis Unit, Roswell Park Cancer Center, Buffalo, New York 14203, USA

*Corresponding author: Anwarul Islam, Clinical Associate Professor of Medicine, Division of Hematology/Oncology, Department of Medicine, Buffalo General Hospital, Buffalo, New York 14203, USA

\section{ARTICLE INFO}

Received: April 08, 2020

Published: 幽 April 23, 2020

Citation: Anwarul Islam, Joanne Becker. A Case of Smoldering Multiple Myeloma with Simultaneous Increase of a Polyclonal Immunoglobulin. Biomed J Sci \& Tech Res 27(2)-2020. BJSTR. MS.ID.004468.

\begin{abstract}
The majority of patients with multiple myeloma (monoclonal gammopathies) typically have a single clone of plasma cells, secreting a unique type of monoclonal protein, one heavy chain and one light chain. In a small proportion of patients, more than one (usually two) types of monoclonal proteins can be seen and they may differ with respect to heavy chain or light chain or both. But a simultaneous presence of monoclonal and polyclonal protein in the same case is rare. We present a case whose serum protein electrophoresis revealed two spikes in gammaglobulins and immunofixation electrophoresis identified two protein components, one- IgA kappa only (monoclonal) and one IgG kappa+lambda (polyclonal). The association and the significance of the presence of polyclonal immunoglobulin in multiple myeloma is discussed.
\end{abstract}

Keywords: Multiple Myeloma; Smoldering Multiple Myeloma; Monoclonal Gammopathy; Polyclonal Gammopathy

\section{Case Report}

The patient, an 86 years old white female was referred for the evaluation and management of anemia and protein abnormalities. The patient did not have any major complaints except for back pain and pain in her right hip which was replaced three times. Her past medical history was significant for arthritis, GERD, diverticulitis, macular degeneration, and a minor stroke. She was also diagnosed with lupus syndrome. On physical examination the patient was noted to be mildly anemic but not in acute distress. Her chest was clear to auscultation and vital signs were normal. Laboratory investigations revealed a WBC $4.4 \times 109 / \mathrm{L}$, hemoglobin $10.3 \mathrm{~g} / \mathrm{dL}$ with a normal MCV and MCH, and a platelet count of $161 \times 10^{9} / \mathrm{L}$. Her ESR was elevated at $131 \mathrm{~mm} / \mathrm{hr}$. Her CMP was normal except for mildly increased protein at $9.1 \mathrm{~g} / \mathrm{dl}$. Her IgA was raised at $1601.0 \mathrm{mg} / \mathrm{dl}$ (70.0-390.0) and the IgG was high at $2562 \mathrm{mg} / \mathrm{dl}$ (700-1600). IgM was normal at $77 \mathrm{mg} / \mathrm{dl}$ (50-300). Her beta 2 microglobulin was slightly raised at $2.6 \mathrm{mg} / \mathrm{L}(0.8-2.2)$. The serum albumin, calcium and GFR were normal. Serum protein electrophoresis (SPEP) revealed two spikes in gammaglobulins. Serum immunofixation electrophoresis (IFE) revealed IgA kappa (monoclonal) and IgG kappa + lambda polyclonal immunoglobulinemia (Figure 1).

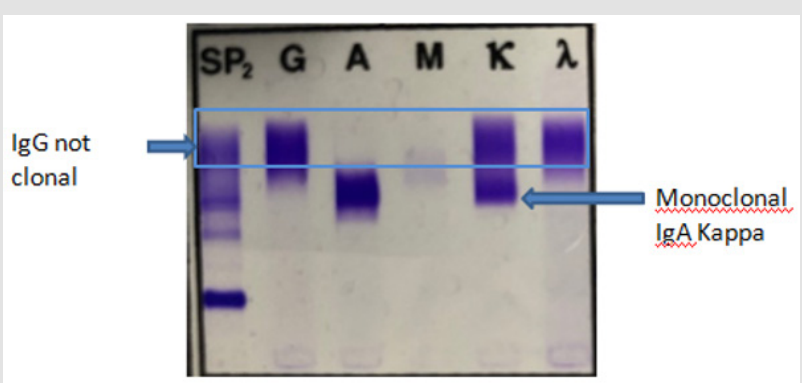

Figure 1: IFE: one band each in IgA and IgG heavy chain lanes with one band in kappa light chain corresponding to IgA heavy chain lane but two bands in kappa and lambda light chain corresponding to IgG heavy chain lane. 
Her 24 hr. urinary Bence Jones protein (BJP) was high at 32.6 (0.00-0.00) and urine IFE revealed kappa BJP. Skeletal survey as well as whole body MRI was negative. A bone marrow aspirate smear, clot section, and core biopsy revealed a hypercellular marrow for her age $(60 \%)$ with maturing trilineage hematopoisis and involvement (10\%) with a plasma cell neoplasm - IgA-kappa monoclonal gammopathy. Flow cytometry: plasma cell gate contained $0.3 \%$ cells which were positive for CD138, CD38, CD19 and kappa-light chain while they were negative for CD20 and CD 56. Cytogenetics revealed a normal female karyotype. Based on the above data a diagnosis of smoldering multiple myeloma with polyclonal gammopathy was established. We believe this is a rare case of a combination of IgA kappa monoclonal and IgG kappa+lambda polyclonal gammopathy (Figure 2).

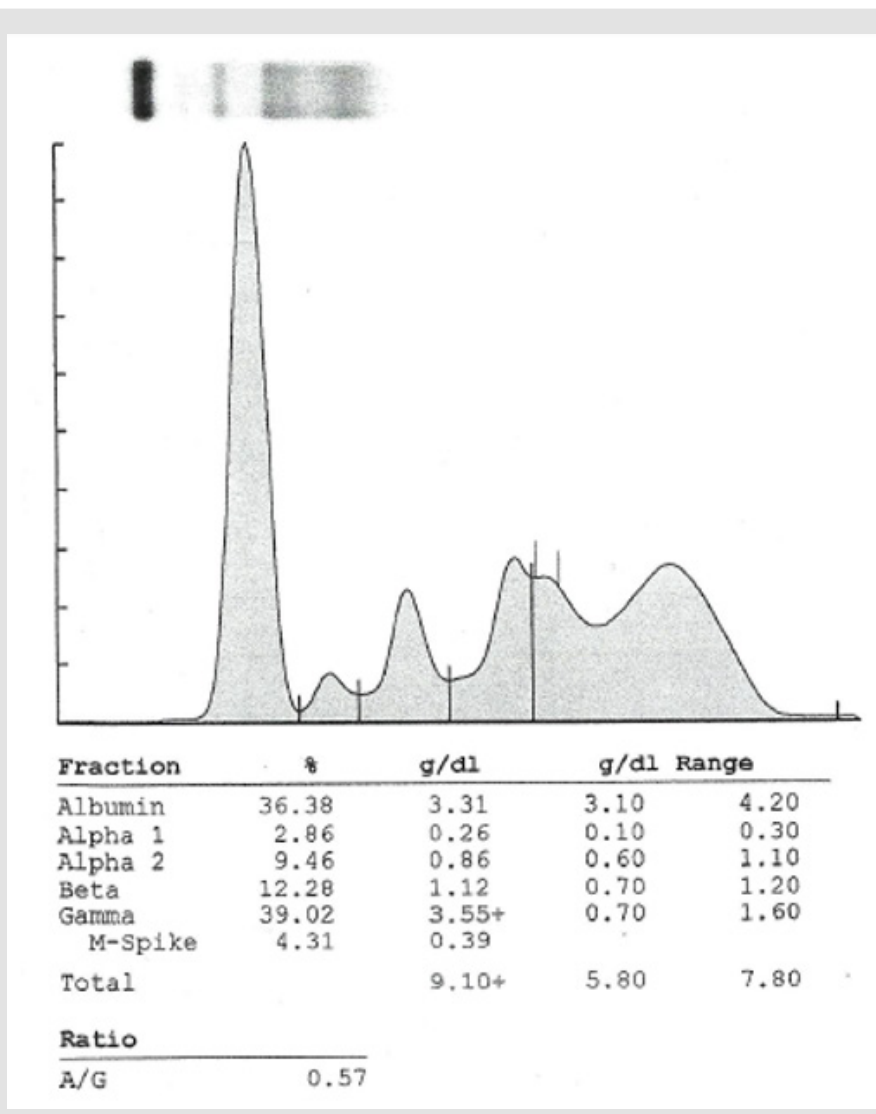

Figure 2: SPE, densitometer tracing.

\section{Discussion}

Multiple myeloma (monoclonal gammopathy) is a group of plasma cell neoplasms which result in the production of a specific and unique monoclonal immunoglobulin (M-component). Biclonal gammopathy is characterized by the simultaneous appearance of two different M- components [1]. The incidence of biclonal gammopathy is rare and constitutes about $1-2 \%$ of all monoclonal gammopathies [2]. The occurrence of gammopathies characterized by simultaneous appearance of one monoclonal and one polyclonal protein is rare. In this case the monoclonal gammopathy (IgA kappa) may have resulted in the neoplastic transformation of a cell clone undergoing immunoglobulin class switching or due to an independent neoplastic transformation event yielding a proliferation of unrelated plasma cell clone [3]. The associated polyclonal gammopathy (IgG kappa+lambda) of the present patient may be a reflection of her underlying conditions such as arthritis and lupus. A polyclonal increase of immunoglobulins has been known to be associated with an inflammatory or reactive process, whereas a monoclonal increase of immunoglobulins usually results from a neoplastic proliferation of plasma cells [4]. Based on the clinical and laboratory features monoclonal gammopathies can be classified into four groups: monoclonal gammopathy of undetermined significance (MGUS), smoldering multiple myeloma (SMM), multiple myeloma and monoclonal gammopathy associated lymphoproliferative disease including lymphoma, macrogloblinemia, chronic lymphocytic leukemia and unclassified lymphoproliferative disorder.

Most cases of smoldering multiple myeloma remain stable and rarely develop symptomatic multiple myeloma after several years of follow-up. However, the significance of simultaneous presence of increased levels of polyclonal immunoglobulin in the presence monoclonal protein remains unclear but it may not actually affect the prognosis or outcome of the disease. Although the patient's serum showed a monoclonal spike but her M protein was less $3 \mathrm{~g} / \mathrm{dl}$, the bone marrow plasma cells represented $10 \%$ of the hemopoietic cells and there were no myeloma defining events. The patient was 
thus considered to have smoldering multiple myeloma as per IMWG criteria [5]. It has been reported that polyclonal gammopathy with immunoglobulin levels exceeding the normal range associated with polyclonal plasmacytosis occurs with relatively high frequency in patients treated with prolonged course of lenalidomide [6]. This was unlikely to be the case in our patient as she was never adminstered lenalidomide. In the present case the presence of polyclonal gammopathy (polyclonal increase of gamma globulin) is most likely to be due to the presence of an underlying condition such as an inflammatory or reactive process or auto-immune disorder such as lupus syndrome [7]. Although there is limited information on the clinical course of patients with simultaneous presence of monoclonal and polyclonal gammopathies, we believe an accurate diagnosis of polyclonal gammopathies in association with MGUS, Smoldering multiple myeloma and multiple myeloma may have implications on the disease manifestation, progression, prognosis and response to therapy.

\section{References}

1. Kyle RA, Robinson RA, Katzmann JA (1981) The clinical aspects of biclonal gammopathies. Review of 57 cases 71(6): 999-1008.

ISSN: $2574-1241$

DOI: 10.26717/BJSTR.2020.27.004468

Anwarul Islam. Biomed J Sci \& Tech Res

(C) This work is licensed under Creative

Submission Link: https://biomedres.us/submit-manuscript.php
2. Banerjee A, Pimpalgaonkar K, Christy AL (2016) A Rare Case of Multiple Myeloma with Biclonal Gammopathy. Clin Diagn Res 10(12): BD03BD04.

3. Yadov A, Reddy R, Malathi M (2017) Multiple Myeloma with Biclonal Gammopathy of IgA Kappa Variant: A Case Report. Biochem Anal Biochem 6: 4.

4. Pradhan D, Arora P, Gami A, Neeraj Kaur (2015) Immunoglobulin G kappa biclonal gammopathy associated with multiple myeloma, plasmacytoma and cast nephropathy. E-JCRT Correspondence 11(3): 660.

5. Srinivasan VK, Bhagat P, Bansal F, Seema Chhabra (2016) Occurrence of Double Monoclonal Bands on Protein Electrophoresis: An Unusual Finding. Indian J Hematol Blood Transfus 32(1): 184-188.

6. Rajkumar SV, Dimopoulos MA, Palumbo A, Joan Blade, Giampaolo Merlini, et al. (2014) International Myeloma Working Group updated criteria for the diagnosis of multiple myeloma. Lancet Oncol 15(12): e538-e548.

7. Zamarin D, Devlin S, Arcila M, H Landau, A Lesokhin, et al. (2012) Reactive Polyclonal Gammopathy Associated with Polyclonal Plasmacytosis Is Common in Patients with Multiple Myeloma Receiving Prolonged Lenalidomide Therapy: A Retrospective Study of 104 Patients. Blood 12(21): 4033

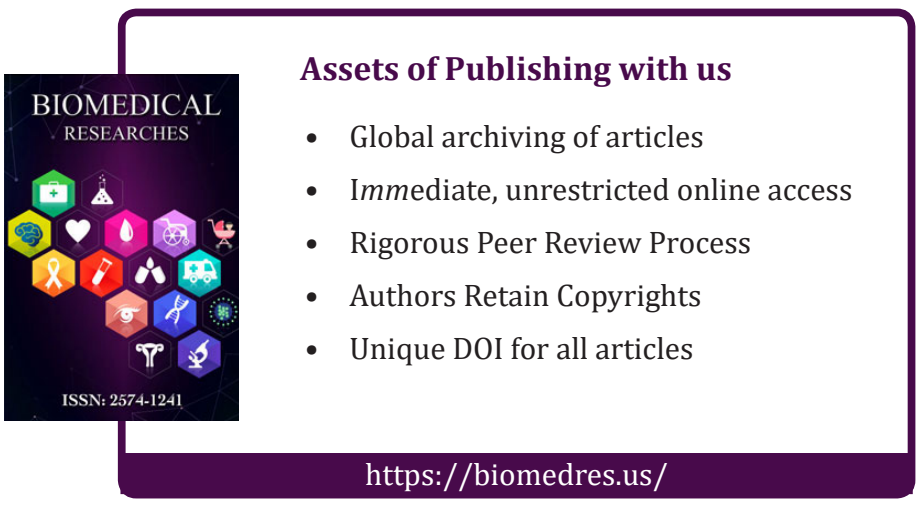

